\title{
Avaliação do Impacto da Corticoterapia Antenatal para Aceleração da Maturidade Pulmonar Fetal nos Recém-nascidos em Maternidade-Escola Brasileira
}

\author{
Impact of Antenatal Corticosteroid Therapy for the Acceleration of Fetal Lung \\ Maturation in Neonates at a Teaching Hospital in Brazil
}

Isabela Cristina Coutinho de Albuquerque, Melania Maria Ramos de Amorim, Jucille Meneses, Leila Katz, Luiz Carlos Santos

\section{RESUMO}

Objetivos: avaliar os efeitos da corticoterapia antenatal na incidência da sindrome do desconforto respiratório do recém-nascido (SDRN), outras morbidades e óbito em neonatos prematuros atendidos em maternidade-escola (IMIP) no Brasil.

Métodos: realizou-se estudo analítico, observacional, tipo coorte, analisando a evolução de 155 recém-nascidos (RN) de mulheres internadas no IMIP com parto prematuro, sendo que 78 receberam corticóide e 77 não receberam, verificando-se o esquema utilizado, a incidência de SDRN, e outras morbidades associadas com prematuridade e morte neonatal, entre fevereiro e novembro de 2001. Determinou-se a razão de risco e seu intervalo de confiança a 95\% para SDRN e os diversos desfechos neonatais (variáveis dependentes), de acordo com o uso ou não de corticóide antenatal (variável independente).

Resultados: a corticoterapia foi administrada a 50,3\% das pacientes $164 \%$ receberam esquema completo e $36 \%$ esquema incompleto). A incidência de SDRN foi significantemente menor entre os $R N$ cujas mães receberam corticóide $(37,2 \%)$ em relação às que não receberam (63,6\%). Não houve redução no risco das morbidades associadas à prematuridade. Verificou-se redução no risco de morte (39\%) e na freqüência de oxigenoterapia (37\%), sem diferença no tempo de oxigenoterapia ou de hospitalização. Após análise de regressão logística múltipla, observou-se redução no risco de SDRN de $72 \%$ para o uso de corticóide e aumento de sete vezes neste risco para os $R N$ com idade gestacional menor que 32 semanas.

Conclusões: verificou-se impacto favorável da corticoterapia antenatal, com redução significativa da SDRN na idade gestacional entre 26 e 35 semanas. Embora não tenha se verificado redução de outras morbidades, isso pode ter sido devido ao pequeno tamanho da amostra.

PALAVRAS-CHAVE: Prematuridade. Sindrome do desconforto respiratório do recém-nascido.

\section{Introdução}

A prematuridade constitui a principal causa de morbimortalidade neonatal, não tendo sido

Instituto Materno - Infantil de Pernambuco (IMIP) - Recife - PE

Correspondência:

Melania Maria Ramos de Amorim

Rua Neuza Borborema de Sousa, 300 - Bairro Santo Antônio

58103-313 - Campina Grande - PB

e-mail: melamorim@uol.com.br observado o seu declínio até o momento, apesar do uso de múltiplos agentes farmacológicos ${ }^{1}$. Metanálises de ensaios clínicos randomizados indicam que o tratamento tocolítico pode prolongar a gravidez por 48 a 72 horas, mas não reduzir a incidência de parto pré-termo ou a morbimortalidade perinatal. A sua maior efetividade deriva de cessação das contrações uterinas e retardo temporário do parto, contribuindo para melhora no resultado neonatal, dando oportunidade para que 
os partos ocorram em centros capazes de dar o suporte necessário a recém-nascido pré-termo, além de permitir a administração de corticosteróides, para reduzir o risco da síndrome do desconforto respiratório do recém-nascido (SDRN) e outras complicações neonatais ${ }^{2}$.

A SDRN está associada com a deficiência de surfactante pulmonar e representa séria complicação da prematuridade, causando significativa morbidade e mortalidade em curto e longo prazo, contribuindo substancialmente para elevados custos dentro das unidades de cuidados intensivos neonatais ${ }^{3}$. Sua importância persiste ainda em nossos dias, apesar dos grandes avanços nesses cuidados intensivos e, sobretudo, da introdução da terapia com surfactante exógeno. Outras medidas que contribuíram para o declínio da SDRN foram a introdução da ventilação mecânica, e principalmente, o aumento do uso de esteróides no período antenatal ${ }^{4}$.

Com o aumento da taxa de sobrevivência dos recém-nascidos pré-termo afetados por SDRN, têmse observado que diversas outras entidades nosológicas, principalmente a displasia broncopulmonar, o suporte ventilatório pulmonar por longo período, a enterocolite necrosante e complicações neurológicas, como a hemorragia intraperiventricular e a leucomalácia periventricular, contribuem significativamente para as sérias morbidades experimentadas pelos prematuros ${ }^{5}$. Por outro lado, a corticoterapia antenatal reduz a incidência e a gravidade destas morbidades ${ }^{6}$.

É possível que a falta de níveis adequados de corticóide contribua para adaptação respiratória inadequada em recém-nascidos pré-termo, uma vez que a concentração desta substância se encontra baixa nestes neonatos, e permanece baixa após o parto, mesmo que eles sejam expostos a fatores de estresse ${ }^{7}$. Os prováveis mecanismos de ação dos corticosteróides incluem o aumento da produção ou liberação de surfactante e a absorção acelerada de líquido pelos pulmões, ambos influenciados pelo nivel de corticóide endógeno ao ter$\mathrm{mo}^{8}$. Os corticosteróides empregados para a promoção da maturação pulmonar fetal são aqueles capazes de atravessar a barreira placentária, sendo a sua forma de administração por via intramuscular (IM) na dose de $24 \mathrm{mg}$ (tanto betametasona quanto dexametasona).

Em 1994, o Instituto Nacional de Saúde dos Estados Unidos publicou a declaração consensual dos efeitos do corticóide para a maturação fetal nos resultados perinatais, recomendando "a utilização da corticoterapia antenatal para mulheres com risco de parto pré-termo, salvo poucas exceções, podendo resultar em substancial decrésci- mo na mortalidade e morbidade neonatal, assim como em importante economia nos custos dos cuidados de saúde". Afirmou-se ainda, nesse texto, que a corticoterapia neonatal constitui "raro exemplo de uma tecnologia que traz importante economia além da promoção de saúde" 9 .

Uma vez comprovados os efeitos benéficos conferidos pela corticoterapia antenatal em relação à incidência da SDRN e considerando que o seu uso faz parte da rotina estabelecida para as mulheres que estão sob o risco de parir prematuramente na maternidade do IMIP, decidimos realizar este trabalho, na tentativa de avaliar o impacto desta intervenção sobre a incidência de SDRN e outras morbidades da prematuridade.

\section{Métodos}

Realizou-se estudo observacional, tipo coorte, incluindo 155 casos de parto prematuro atendidos no Instituto Materno-Infantil de Pernambuco (IMIP) - Recife/PE, no período de fevereiro a novembro de 2001. O projeto deste estudo foi aprovado pelo Comitê de Ética em Pesquisa do IMIP. A coleta de dados foi realizada prospectivamente por um dos pesquisadores, atendo-se às definições apresentadas a seguir.

Os critérios de inclusão foram: gestação única e tópica, concepto vivo e idade gestacional entre 26 e 35 semanas. Foram excluídos os casos de malformações congênitas, infecção congênita comprovada, pneumonia por Streptococcus $\beta$ haemolyticus do grupo $\mathrm{B}$, eventos hemorrágicos maternos agudos e intensos que pudessem comprometer o bem-estar fetal e uso de corticosteróides para tratamento de doenças crônicas durante a gravidez. No periodo mencionado, foram assistidos no IMIP 1149 partos prematuros (27\% do total de partos atendidos na instituição), dos quais apenas 155 preencheram os critérios de inclusão e exclusão.

A variável independente foi a administração de corticóide antenatal, sendo utilizada a betametasona $(12 \mathrm{mg}$ IM, repetindo-se com 24 horas), conforme a rotina da instituição. Foram incluídas na análise tanto as pacientes com ciclo completo como incompleto (apenas uma dose). Como variáveis dependentes foram analisadas: SDRN, hemorragia intra e periventricular, enterocolite necrosante, persistência do ducto arterioso e sepse neonatal, utilização e tempo de oxigenoterapia, duração da hospitalização e morte neonatal.

Para determinação acurada da idade gestacional, consideraram-se parâmetros clínicos (data 
da última menstruação conhecida e confiável), ecográficos (ultra-sonografia) e a avaliação do recém-nascido pelo método de Ballard et al. ${ }^{10}$, admitindo-se discrepância entre os métodos de no máximo uma semana, dentro dos limites previstos para o estudo (26 a 35 semanas). Como a maioria das gestantes não tinham se submetido a ultrasonografia no primeiro trimestre, considerou-se a ultra-sonografia realizada em idade gestacional mais precoce para o cálculo da idade gestacional corrigida.

A sindrome do desconforto respiratório do recém-nascido foi definida segundo os critérios utilizados pelo berçário do IMIP, que consistem na ocorrência de desconforto respiratório precoce e progressivo com alterações radiológicas típicas e os achados clínicos de taquipnéia, gemido expiratório, tiragem intercostal e subcostal, batimentos de asas de nariz e ausculta pulmonar com murmúrio vesicular diminuído ${ }^{11}$. A hemorragia intraperiventricular representa o tipo de hemorragia intracraniana mais freqüente entre os recém-nascidos pré-termo, tendo sido classificada segundo os critérios de Papile et al. ${ }^{12}$. Para o diagnóstico da hemorragia, um exame ultra-sonográfico transfontanela foi realizado em todos os recém-nascidos prematuros. Considerou-se displasia broncopulmonar a dependência de oxigênio aos 28 dias de vida, com alterações radiológicas típicas e passado de ventilação mecânica ou oxigenoterapia $^{13}$. Por meio da ecodopplercardiografia, realizada apenas nos casos com suspeita clínica, definiu-se persistência do ducto arterioso quando presente shunt esquerda-direita (entre a aorta e a artéria pulmonar $)^{14}$. A sepse neonatal foi definida a partir da evidência clínica de processo infeccioso, com sintomas e sinais inespecíficos acompanhados de duas ou mais alterações laboratoriais, conforme a rotina estabelecida pelo Serviço $^{11}$. Considerou-se como oxigenoterapia a utilização de qualquer uma das técnicas de suporte ventilatório (CPAP nasal, ventilação mecânica assistida ou hallo).

A análise dos dados foi realizada com softwares estatísticos Epi-Info 2000 (de domínio público) e SPSS 8.0 para Windows. Foram utilizados os testes $\chi^{2}$ de associação, $t$ de Student e MannWhitney. Adotou-se o nível de significância de 5\% e a razão de risco com seu intervalo de confiança a 95\% (IC 95\%) como medida do risco relativo das diversas variáveis dependentes. A análise de Mantel-Haenszel foi utilizada para a determinação da razão de risco de SDRN ajustada, controlando-se o efeito do peso ao nascer e da idade gestacional.

A análise de regressão logística múltipla foi usada para determinar as variáveis mais forte- mente associadas à SDRN (variável dependente). As variáveis independentes incluídas no modelo foram: idade gestacional (menor que 32 semanas / maior ou igual a 32 semanas), uso de corticóide ( $\operatorname{sim} /$ não), pré-eclâmpsia ( $\operatorname{sim} /$ não), trabalho de parto ( $\operatorname{sim} /$ não), tocólise ( $\operatorname{sim} /$ não), oligohidrâmnio ( $\operatorname{sim} /$ não), amniorrexe prematura (sim/não), tempo de bolsa rota (em horas), sofrimento fetal crônico (sim/não), tipo de parto (cesárea/parto normal), sexo (masculino/feminino) e Apgar (menor que $7 /$ maior ou igual a 7). Essas variáveis foram codificadas binariamente, exceto o tempo de bolsa rota (variável contínua). Adotou-se o comando retrógrado para regressão condicional, com análise passo a passo, em que as variáveis que não contribuíam significativamente ( $>0.05)$ eram eliminadas a cada passo. Calculou-se assim o risco ajustado de SDRN para as variáveis que persistiram no modelo.

\section{Resultados}

Das 155 mulheres envolvidas no estudo, 77 $(49,7 \%)$ não receberam e $78(50,3 \%)$ receberam o corticóide (betametasona), sendo as principais indicações o trabalho de parto prematuro $(61,3 \%)$, pré-eclâmpsia $(43,2 \%)$, amniorrexe prematura $(34,8 \%)$, oligo-hidrâmnio $(17,5 \%)$ e sofrimento fetal crônico $(15,4 \%)$. Entre as pacientes que receberam corticóide, 50 receberam o esquema completo (dose inicial de $12 \mathrm{mg}$ da betametasona, repetindo-se com 24 horas) e 28 o esquema incompleto (dose única de betametasona). Não houve diferença significante entre as características maternas ou neonatais. Observou-se, contudo, uma maior freqüência de RN com peso menor que 1000 gramas no grupo que recebeu corticóide (Tabela 1).

A incidência de SDRN foi significativamente menor entre as pacientes que receberam corticóide $(37,2$ versus 63,6\%). A análise estratificada por subgrupos de peso evidenciou menor incidência de SDRN, quanto maior o peso dorecém-nascido, e menor freqüência de SDRN com o uso do corticóide em todos os estratos de peso. O risco de SDRN ajustado por peso dos recém-nascidos cujas mães receberam corticóide foi de 0,50 (IC 95\%=0,36-0,70). Por outro lado, a análise estratificada por subgrupos de idade gestacional evidenciou menor incidência de SDRN, quanto maior foi a idade gestacional. Não houve diferença entre o risco bruto e o ajustado de SDRN, mantendose redução de $42 \%$ com a utilização de corticóide antenatal. 
Tabela 1 - Principais características maternas e neonatais de acordo com a utilização ou não de corticóide.

\begin{tabular}{|c|c|c|c|}
\hline \multirow[b]{2}{*}{ Características maternas } & \multicolumn{2}{|c|}{ Uso de corticóide } & \multirow[b]{2}{*}{$p$} \\
\hline & $\begin{array}{c}\text { Sim } \\
n=78\end{array}$ & $\begin{array}{c}\text { Não } \\
\mathrm{n}=77\end{array}$ & \\
\hline Idade materna em anos $(\bar{x} \pm D P)$ & $24,6 \pm 6$ & $23,8 \pm 6$ & 0,4 \\
\hline Idade gestacional em semanas $(\bar{x} \pm D P)$ & $32,1 \pm 1,9$ & $32,1 \pm 1,9$ & 0,9 \\
\hline \multicolumn{4}{|l|}{ Diagnóstico obstétrico } \\
\hline \% Pré-eclâmpsia & 55,1 & 31,2 & 0,02 \\
\hline$\%$ Trabalho de parto prematuro & 51,3 & 71,4 & 0,01 \\
\hline \multicolumn{4}{|l|}{ Características neonatais } \\
\hline$\%$ sexo masculino & 46,2 & 50,6 & 0,57 \\
\hline Peso $<1.000$ gramas & 63,6 & 36,4 & \\
\hline Peso entre $1.000-1.499$ gramas & 55,0 & 45,0 & 0,3 \\
\hline Peso entre $1.500-1.999$ gramas & 43,1 & 56,9 & \\
\hline Peso $\geq 2.000$ gramas & 40,9 & 59,1 & \\
\hline Idade gestacional $\leq 28$ semanas & 3,8 & 2,7 & \\
\hline Idade gestacional entre $29-30$ semanas & 15,4 & 13,3 & 0,9 \\
\hline Idade gestacional entre $31-32$ semanas & 30,8 & 33,3 & \\
\hline Idade gestacional $\geq 33$ semanas & 50 & 50,7 & \\
\hline
\end{tabular}

$\mathrm{Na}$ análise de regressão logística múltipla as variáveis que permaneceram fortemente associadas com a SDRN foram a utilização do corticóide, com redução do risco de $72 \%$, e a idade gestacional inferior a 32 semanas, que aumentou o risco de SDRN em quase sete vezes (Tabela 2). Esse modelo predisse corretamente $83 \%$ dos casos de SDRN.

Não se observou redução do risco das diversas morbidades relacionadas à prematuridade no grupo dos neonatos cujas mães receberam corticóide (Tabela 3). Por outro lado, houve significativa redução (37\%) na necessidade de oxigenoterapia com o uso do corticóide, embora não tenha sido encontrada diferença estatisticamente significante na duração da oxigenoterapia.

\section{Discussão}

Numerosas publicações têm ressaltado o importante papel da corticoterapia na redução da morbimortalidade neonatal ${ }^{15}$, refletindo a expressiva quantidade de evidências científicas que vêm se acumulando ao longo das últimas décadas no campo da perinatologia. A utilização de corticóide antenatal foi amplamente apoiada pelo consenso publicado pelo "National Institutes of Health (NIH)", nos Estados Unidos, em $1994^{9}$.

Tabela 2 - Risco relativo ajustado de SDRN de acordo com os fatores potencialmente confundidores (modelo de regressão logística).

\begin{tabular}{lccrr}
\hline Variável & Coeficiente & Erro-padrão do coeficiente & Risco ajustado & IC 95\% \\
\hline IG $<32$ sem & 1,90 & 0,43 & 6,70 & $5,86-7,54$ \\
Corticóide & $-1,26$ & 0,37 & 0,28 & $0,42-0,98$ \\
Constante & 0,12 & 0,26 & & \\
\hline
\end{tabular}

IG: idade gestacional, IC 95\%: intervalo de confiança a 95\%

Tabela 3 - Incidência das principais morbidades relacionadas à prematuridade e morte neonatal, de acordo com a utilização ou não de corticóide.

\begin{tabular}{|c|c|c|c|c|c|c|}
\hline & \multicolumn{2}{|c|}{$\begin{array}{l}\text { Com corticóide } \\
\qquad(\mathrm{n}=78)\end{array}$} & \multicolumn{2}{|c|}{$\begin{array}{l}\text { Sem corticóide } \\
\qquad(\mathrm{n}=77)\end{array}$} & \multirow[t]{2}{*}{ RR (corticóide) } & \multirow[t]{2}{*}{ IC $95 \%$} \\
\hline & $\mathrm{n}$ & $\%$ & $\mathrm{n}$ & $\%$ & & \\
\hline EN & 1 & 1,3 & 0 & 0 & & \\
\hline DBP & 2 & 2,6 & 1 & 1,3 & 1,92 & $0,17-20,7$ \\
\hline Sepse & 22 & 28,2 & 24 & 32 & 0,88 & $0,54-1,43$ \\
\hline Morte & 10 & 12,8 & 16 & 20,8 & 0,61 & $0,29-1,27$ \\
\hline
\end{tabular}


Considerando-se que todas as mulheres com risco de parto prematuro devem receber tratamento com corticóide, depreende-se que suas indicações incluem gama expressiva de situações clínicas $^{9}$. Além do trabalho de parto prematuro, estas situações abrangem entidades tão diversas como pré-eclâmpsia, amniorrexe prematura, placenta prévia, isoimunização $\mathrm{Rh}$, malformações fetais, restrição do crescimento intra-uterino e diversas outras indicações, tanto maternas como fetais ${ }^{16}$. Convém ainda ressaltar que os beneficios conferidos pelo corticóide antenatal estendem-se a ampla faixa de idade gestacional, compreendido entre 24 e 34 semanas de gestação.

No presente estudo a freqüência de utilização de corticóide foi de apenas $50,3 \%$, sendo que as gestantes que não receberam corticóide encontravam-se, em sua maioria (cerca de $70 \%$ ), com trabalho de parto avançado, o que pode explicar o fato de a não administração do corticóide ter sido devida à falta de intervalo de tempo suficiente, sendo também provável que os obstetras tenham optado por não realizar a tocólise, supondo que não seria possivel a inibição do parto prematuro. Mesmo assim, é importante destacar que a tocólise deveria sempre ser tentada, salvo em condições de parto iminente, porque o objetivo maior da terapia tocolítica não é evitar o parto prematuro, mas postergá-lo por tempo suficiente para que o corticóide exerça a sua ação. A finalidade básica é prevenir não a prematuridade, porém as complicações do recém-nascido prematuro ${ }^{2}$.

Partindo de observações preliminares sobre a obtenção do mesmo beneficio em relação à SDRN com uma única dose de corticóide, após a realização de exaustivos estudos, em agosto de 2000, foi publicado um Consenso pelo $\mathrm{NIH}^{17}$, preconizando a utilização da dose única de corticóide, baseado nos dados que suportam inequivocamente a sua eficácia em relação à redução da incidência da SDRN (50\%) e à inconsistência do uso rotineiro de doses repetidas em relação aos benefícios e riscos, sendo prudente evitar o seu uso na prática clínica.

Acreditamos que não deveriamos nos abster de tomar algumas medidas para postergar o parto prematuro, lançando mão da tocólise, mesmo que esta atitude possa, inicialmente, parecer tentativa infundada e sem sucesso provável. Salvo situações em que a ultimação do parto realmente se faça necessária, como em situações de risco materno ou fetal, todos os esforços devem ser desprendidos para postergar o nascimento e conseguir administrar o corticóide. Não raras vezes, o obstetra se depara com situações surpreendentes, tais como uma parada ou evolução mais arrastada do trabalho de parto, permitindo, caso seja administrado o corticóide, que ele desempe- nhe a sua função. Mesmo em casos de préeclâmpsia grave e até mesmo eclâmpsia e sindrome HELLP, quando a condição clínica materna é estável e não há sofrimento fetal, justifica-se adiar a interrupção da gravidez para permitir a administração da corticoterapia ${ }^{18}$.

Observou-se em nosso estudo que o maior percentual de administração de corticóide antenatal foi encontrado entre os recém-nascidos de mais baixo peso ( $<2.000$ gramas), refletindo de maneira indireta a idade gestacional mais precoce. Na verdade, entre os recém-nascidos envolvidos nesse estudo, $53 \%$ tinham um peso menor do que 1.500 gramas, sendo a maior concentração de prematuros no subgrupo entre 1.000 e 1.499 gramas (39\% dos prematuros). Destacamos inclusive que, entre os prematuros com menos de 1.000 gramas, todos os que não receberam corticóide morreram, ao passo que a mortalidade entre os que receberam corticóide foi de $60 \%$, ainda elevada, porém já apontando para um efeito importante da corticoterapia na sobrevivência em recém-nascidos de muito baixo peso. Na verdade, a incidência de recém-nascido de muito baixo peso foi bastante elevada neste estudo, uma vez que o IMIP constitui hospital de referência, recebendo muitas gestantes sob risco de parto prematuro, em idade gestacional bastante precoce.

Observou-se ainda neste estudo redução significativa da necessidade de oxigenoterapia, mas não no seu tempo de duração, podendo este fato ser explicado pelo perfil de extrema prematuridade dos pacientes atendidos no IMIP, prolongando o tempo de requerimento de suporte ventilatório. Outra explicação é que, com a terapia intensiva neonatal, consegue-se a sobrevivência de recémnascidos que, anteriormente, não sobreviviam ao período crítico das primeiras 72 horas, e assim, prolonga-se a necessidade de oxigenoterapia.

O presente estudo teve algumas limitações que precisam ser consideradas. Como se trata de estudo observacional, não é o ideal para verificar a eficácia de uma droga (no caso o corticóide), porque o desenho de estudo ideal seria um ensaio clínico. No entanto, seria extremamente antiético, no atual estágio de conhecimento, realizar ensaio clínico comparando grupos recebendo ou não o corticóide, uma vez que a eficácia da corticoterapia já foi muitas vezes demonstrada.

Outro fator importante que merece menção foi o número insuficiente de casos (já que o cálculo de nossa amostra foi feito com base no risco de redução da incidência de SDRN), para avaliar eventos mais raros (com respeito às morbidades relacionadas com a prematuridade). Na verdade, para avaliar o verdadeiro impacto da corticoterapia antenatal na redução das outras morbidades relacionadas 
com a prematuridade em nosso meio, seriam necessários novos estudos, com maior tamanho amostral, considerando-se para seu cálculo a freqüência que aqui descrevemos, até então desconhecida (variando entre 1-6\% para cada uma das morbidades). Por outro lado, os rígidos critérios de inclusão e exclusão, necessários para a obtenção de um grupo de prematuros com correta determinação da idade gestacional e sem fatores confundidores que pudessem influenciar os resultados, restringiram em demasia o número de casos estudados, de forma que um estudo mais abrangente teria de, obrigatoriamente, estenderse por um maior período de tempo.

Cumpre destacar que esse estudo foi realizado basicamente em função da necessidade de determinar os resultados da corticoterapia antenatal em nossa Instituição, tentando constatar, na prática, os resultados da adoção de rotinas baseadas em evidências científicas sólidas. Devemos ainda salientar que a avaliação dos próprios resultados faz parte dos cinco passos recomendados para a prática da Medicina Baseada em Evidências, sendo fundamental que os diversos serviços gerem essas evidências ${ }^{19}$.

Vale salientar que nossos resultados vêm apenas corroborar as conclusões dos diversos ensaios clínicos e metanálises já publicados ${ }^{3}$. Reforçamos que, à luz dos conhecimentos atuais, não se pode conceber, nos dias de hoje, que a terapia tocolitica seja prescrita sem vir acompanhada, logo em seguida, da prescrição de corticóide antenatal. O real papel da tocólise é apenas o de postergar o parto prematuro por 24 a 72 horas e permitir a utilização da corticoterapia.

O medo, muitas vezes superestimado, da utilização do corticóide deve ser substituído pelo temor das importantes complicações da prematuridade, com todas as implicações e seqüelas a longo prazo, que podem marcar para sempre a vida futura do recém-nascido prematuro.

\section{ABSTRACT}

Purpose: to evaluate the effects of antenatal corticosteroid treatment on the incidence of respiratory distress syndrome $(R D S)$, neonatal morbidities, and mortality in preterm babies assisted at IMIP, a teaching hospital in Brazil.

Methods: this was an observational, analytical, cohort study which included 155 newborns from women who delivered prematurely. The study was conducted between February and November 2001 and included 78 women in the corticosteroid-treated group and 77 in the nontreated group. The study design included the incidence of RDS, assessment of morbidities related to prematurity and tabulation of neonatal mortality. The risk ratio and its $95 \%$ confidence interval were determined for estimation of the relative risk for RDS and neonatal outcome (dependent variables) according to antenatal corticoid therapy administration (independent variable).

Results: corticosteroid treatment was administered to $50.3 \%$ of the patients $164 \%$ of the women received the full treatment course, while $36 \%$ of the same group received a partial course of treatment). The incidence of RDS was significantly lower in the corticosteroid treated group (37.2\%) compared with the nontreated group (63.6\%). There was no observable decrease in the risk for morbidities associated with prematurity. There was a decrease in mortality and in the frequency of supplemental oxygen therapy in the corticosteroid group (37\%). On multiple logical regression analysis, there was a $72 \%$ reduction in the risk for RDS in the corticosteroid group, and approximately a seven times greater risk for RDS in babies of gestational age below 32 weeks.

Conclusions: a favorable impact of antenatal corticosteroid administration was observed, with significant reduction of the risk for RDS in patients with gestational age between 26 and 35 weeks. Although no effect on the other morbidities was observed, this can be explained by the small size of the sample.

KEY WORDS: Antenatal corticosteroid. Prematurity. Newborn. Respiratory distress syndrome of the newborn.

\section{Referências}

1. Romero R, Sibai BM, Ramos LS, et al. An oxytocin receptor antagonist (atosiban) in the treatment of preterm labor: a randomized, double-blind, placebocontrolled trial with tocolytic rescue. Am J Obstet Gynecol 2000; 182:1173-83.

2. Katz VL, Farmer RM. Controversies in tocolytic therapy. Clin Obstet Gynecol 1999; 42:802-19.

3. Crowley P. Prophylactic corticosteroids for preterm birth. Cochrane Database Syst Rev 2002; (3):CD000065.

4. Lee K, Khoshnood B, Wall SN, Chang Y, Hsieh H, Singh JK. Trend in mortality from respiratory distress syndrome in the United States, 1970-1995. J Pediatr 1999; 134:434-40.

5. Hack MM, Wright LL, Shankaran S, et al. Very low birth weight outcomes of the National Institute of Child Health and Human Development Neonatal Network, November 1989 to October 1990. Am J Obstet Gynecol 1995; 172:457-64.

6. Ballard RA, Ballard PL. Antenatal hormone therapy for improving the outcome of the preterm infant. J Perinatol 1996; 16:390-6.

7. al Saedi S, Dean H, Dent W, Cronin C. Reference ranges for serum cortisol and 17- hydroxyprogesterone levels in preterm infants. J Pediatr 1995; 126:985-7. 
8. Barker PM, Gowen CW, Lawson EE, Knowles, MR. Decreased sodium ion absorption across nasal epithelium of very premature infants with respiratory distress syndrome. J Pediatr 1997; 130:373-7.

9. Effect of corticosteroids for fetal maturation on perinatal outcomes. NIH Consensus Statement 1994; 12:1-24.

10.Ballard JL, Khoury JC, Wedig K, Wang L, EilersWalsman BL, Lipp R. New Ballard Score, expanded to include extremely premature infants. J Pediatr $1991 ; 119: 417-23$.

11.Lima G, Duque T, Meneses J. Pautas de neonatologia da Unidade Neonatal do IMIP. IMIP; 2000.

12.Papile LA, Burstein L, Burnstein R, Koffler H. Incidence and evolution of subependymal and intraventricular hemorrage: a study of infants with birth weight less than 1,500 gm. J Pediatr 1978; 92:529-33.

13.Jobe AH, Bancalari E. Bronchopulmonary dysplasia. Am J Respir Crit Care Med 2001; 163:1723-9.

14. Clyman RI. Patent ductus arteriosus in the premature infant. In: Ballard RA, Taeusch HW, editors. Diseases of the newborn. $7^{\text {th }}$ ed. Philadelphia: W. B. Saunders; 1998. p.699-707.
15.Vermillion ST, Soper DE, Bland ML, Newman RB. Effectiveness of antenatal corticosteroid administration after preterm premature rupture of the membranes. Am J Obstet Gynecol 2000; 183:925-9.

16.Leviton LC, Goldenberg RL, Baker CS, et al. Methods to encourage the use of antenatal corticosteroid therapy for fetal maturation: a randomized controlled trial. JAMA 1999; 281:46-52.

17.Antenatal corticosteroids revisited: repeat courses NIH Consensus Statement 2000; 17:1-10.

18.Santos LC, Amorim M, Porto AMF, Guimarães V. Obstetrícia: diagnóstico e tratamento (atualização). IMIP; 2000.

19. Sackett DL, Straus SE, Richardson WS, Rosenberg W, Haynes RB. Evidence-Based Medicine. How to practice and teach EBM. $2^{\text {nd }}$ ed. Toronto: Churchill Livingstone; 2000.
Recebido em: 26/9/2002 Aceito com modificações em: 2/ 12/2002

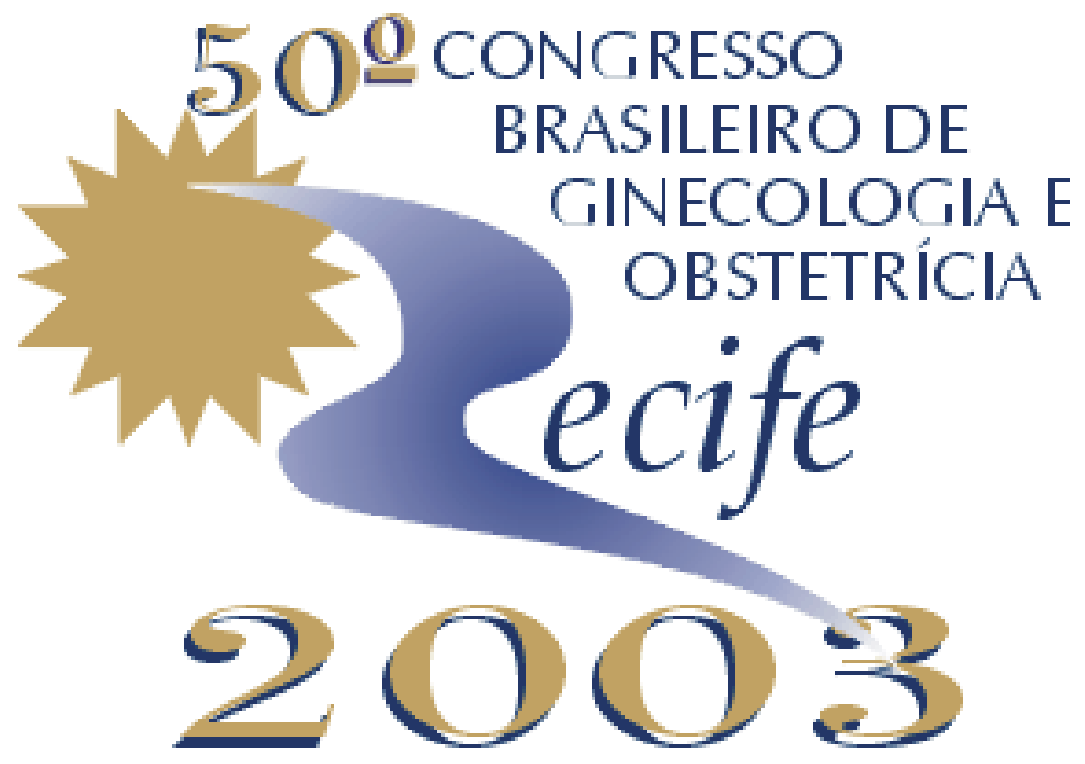

\section{8 a 22 de novembro}

\section{INFORMAÇÓES:}

Telefone: (81) 3222-5112 E-mail: sogope1@terra.com.br 